\title{
DEPOIMENTOS DE MULHERES SOBRE A MENOPAUSA E O TRATAMENTO DE SEUS SINTOMAS
}

\author{
Ana lúcia Valadares, Aarão Mendes Pinto-Neto*, Délio Marques Conde, Maria José Osis, Maria Helena de Sousa, Lúcia Costa-Paiva
}

Trabalho realizado pelo departamento de Tocoginecologia da Universidade Estadual de Campinas, SP

\author{
* Correspondência: \\ Departamento de \\ Tocoginecologia \\ Universidade Estadual \\ de Campinas \\ Rua Alexander Fleming, \\ 101, Cidade Universitária \\ "Zeferino Vaz", \\ CEP 13083-970 - \\ Campinas - SP \\ Tel/Fax: (19) 3521-9306 \\ aarao@unicamp.br
}

\begin{abstract}
RESUMO
Objetivo. Avaliar a percepção de um grupo de mulheres de Belo Horizonte (MG) sobre a menopausa e seu tratamento. Métodos. Realizou-se análise secundária de dados de estudo populacional de corte transversal com 378 mulheres brasileiras natas, de 40 a 65 anos, com II anos ou mais de educação formal. Avaliaram-se os relatos escritos espontaneamente ao final de um questionário sobre sexualidade, entregue por auxiliares de pesquisa e auto-respondido anonimamente. As mulheres foram alocadas em dois grupos: as que escreveram comentários ao final do questionário e as que não escreveram. Compararam-se os grupos em relação às características sociodemográficas e reprodutivas por meio do teste Qui quadrado de Pearson. Os comentários foram transcritos na íntegra para arquivo computadorizado para realizar a análise temática de seu conteúdo, identificando-se e categorizando-se as unidades de significado.

Resultados. Aproximadamente um terço das mulheres escreveu comentários ( I |4/378), o que foi significativamente mais freqüente entre as mulheres com menor renda familiar. As principais categorias de análise identificadas foram: a) insegurança/ confusão, com as principais idéias referentes a angústia, estresse e dúvidas sobre a menopausa; b) sintomas que provocam sentimentos negativos, como ondas de calor, secura vaginal e alterações de humor; c) terapia de reposição hormonal, medos e sentimentos da falta de convicção por parte dos médicos em prescrevê-la.

Conclusão. Os comentários indicam a necessidade de dar mais atenção aos problemas percebidos no climatério, particularmente direcionada às mulheres menos favorecidas economicamente.

Unitermos: Climatério. Terapia de reposição hormonal. Menopausa. Saúde da mulher. Estudos transversais.
\end{abstract}

\section{INTRODUÇÃO}

As conseqüências endócrinas e físicas da diminuição da secreção de esteróides ovarianos são bem conhecidas. Resumidamente, a situação durante os anos de pós-menopausa caracteriza-se pela ausência de progesterona, por concentrações baixas de estrogênios derivados da conversão periférica da testosterona e androstenediona em estrona e, na maioria das vezes, com concentrações menores de androgênios. As conseqüências físicas são, usualmente, um período de instabilidade vasomotora, atrofia dos caracteres sexuais secundários, diminuição da massa óssea e aumento do risco de doenças cardiovasculares!.

As conseqüências psicológicas, ao contrário das conseqüências físicas, são mais difíceis de definir exatamente. Quais das mudanças observadas no comportamento - dinamismo, sensação de bem-estar e libido - devem ser atribuídas somente às alterações hormonais? Quais podem ser explicadas por fatores sociais, pela atitude ante à menopausa, pelo sentimento de estar envelhecendo? Quais das mudanças psicológicas que ocorrem podem ser explicadas como conseqüências dos problemas físicos, isto é, da redução dos níveis hormonais, como sudorese noturna e, conseqüentemente, distúrbios do sono?

O impacto físico e psicológico da deficiência estrogênica pode ser muito mais difícil de ser estabelecido do que se imagina ou se aceita. Existe uma atitude geral em considerar o climatério como fenômeno natural, com tendência a minimizar as conseqüências físicas e psicológicas da deficiência dos esteróides sexuais. A atitude dos profissionais envolvidos nos cuidados de mulheres no climatério varia de acordo com a maneira como se percebe o fenômeno: uma questão natural ou uma enfermidade que ocorre em todas as mulheres. A idéia de um fenômeno natural prevalece, uma vez que a menopausa ocorre em todas as mulheres, geralmente entre os 45 e 55 anos, condição não fatal e para a qual a terapia hormonal está disponível há algumas décadas.

É, pois, lógico considerar as atitudes conservadoras ante à menopausa: ela é inevitável, os sintomas desaparecem espontaneamente em muitas mulheres e naquelas com sintomas intensos e persistentes um novo equilíbrio será alcançado, se necessário com alguma ajuda médica. O significado da menopausa depende também de aspectos culturais, influências sociais e conhecimento pessoal, assim como da interação entre esses fatores ao longo da vida 2,3,4. A auto-imagem é um componente importante que pode associar-se tanto à prevalência quanto à intensidade dos sintomas, bem como à atitude ante à menopausa. Mulheres com baixa auto-estima apresentam muitos sintomas e geralmente têm atitude negativa nesse período da vida ${ }^{3,5,6}$.

Dessa forma, esses fatores podem contribuir para a diminuição da qualidade de vida, assim como levar ao decréscimo da produtividade no trabalho e a dificuldades nos relacionamentos pessoais e sociais ${ }^{4,6}$. 
O período do climatério é freqüentemente visto com preocupação pelas mulheres e correlacionado a problemas e sintomas diversos ${ }^{4,7}$. Segundo Lasnick ${ }^{8}$, quando as mulheres falam das dificuldades ou do sofrimento que experimentam, referem-se essencialmente à crise vivenciada por sua parte feminina, seja no âmbito materno ou na sua capacidade de sedução. Um conhecimento mais amplo sobre 0 climatério a torna mais segura e diminui as crenças negativas em relação à menopausa?. No entanto, os temas femininos ainda encontram entraves para serem veiculados nos meios de comunicação na atualidade, mesmo em revistas dirigidas a esse público, o que pode ser explicado pela questão do gênero ${ }^{10}$.

Estudos que avaliaram os efeitos da educação das mulheres quanto às mudanças de atitudes diante da menopausa e da adesão ao tratamento hormonal relataram que elas se sentiram melhor e que a taxa de continuidade do tratamento hormonal foi maior nas que receberam informações, quando comparadas ao grupo que não recebeu essas orientaçōes. Isso reforça a importância da orientação adequada. Além disso, sabe-se que o uso da terapia hormonal relaciona-se aos níveis socioeconômicos e educacionais elevados ". O fato é que parece haver falta de informação quanto aos riscos e benefícios da terapia hormonal ou de outras formas de tratamento da síndrome do climatério, o que gera dúvidas e inseguranças.

Apesar das pesquisas quantitativas sobre sintomas, aspectos psicossociais durante os anos climatéricos e uso da terapia hormonal, há poucas informações sobre o significado pessoal e a visão da experiência de mulheres brasileiras sobre tais assuntos. O presente artigo avaliou a percepção de um grupo de mulheres sobre a menopausa, seu significado e seu tratamento.

\section{MÉtodos}

Este foi um estudo de corte transversal cujo objetivo principal foi avaliar a sexualidade em mulheres climatéricas. Tratou-se de pesquisa do tipo inquérito populacional, sendo calculado um tamanho amostral necessário de 377 questionários respondidos e concluído com 378 questionários válidos ${ }^{12}$. O tamanho da populaçãa-alvo $(\mathrm{N})$ - população feminina de Belo Horizonte, MG, na faixa etáría entre 40 e 65 anos, com II anos ou mais de educação formal, foi de 44.313 no ano de 2000. Essa informação foi obtida por meio de tabulações especiais dos microdados do Censo Demográfico do Brasil no ano de 2000, realizada pelo Departamento de Demografia - CEDEPLAR da Universidade Federal de Minas Gerais'13. Considerou-se uma proporçãa populacional estimada de mulheres com disfunção sexual de $43 \%{ }^{14}$.

Entrevistadoras treinadas nortearam-se por mapas das regiões sorteadas, foram às residências e verificaram se havia mulheres que preenchiam os critérios de inclusão. Uma vez preenchidos, convidaram essas mulheres a participar da pesquisa. Se elas concordavam, era entregue um questionário para ser respondido. Era agendado um dia para o recolhimento do mesmo. Se elas não se encontravam no local, a abordagem era feita por telefone e, caso quisessem participar, era-lhes enviado o questionário. A pesquisadora principal telefonava para cada uma das participantes e confirmava se o questionário estava respondido. Em caso positivo, o mesmo era recolhido por mensageiro em envelope sem identificação e colocado em caixa de correio lacrada ${ }^{2}$.
O questionário do estudo continha perguntas sobre sexualidade ${ }^{15}$, dados sócio-demográficos, comportamentais e relativos à saúde. Além disso, uma seção desse instrumento tinha como objetivo classificar o estrato socioeconômico, de acordo com os critérios de classificaçãao econômica do Brasil|'

Para este artigo, foram avaliados relatos espontâneos das mulheres quanto à sua percepção sobre a menopausa e seu tratamento. Esses relatos foram escritos ao final do questionário respondido anonimamente, em espaço aberto deixado para que cada mulher, que assim o desejasse, manifestasse espontaneamente suas opiniões sobre os temas abordados.

Para a análise apresentada neste artigo, inicialmente as mulheres foram classificadas em dois grupos: as que escreveram comentários ao final do questionário e as que não escreveram; e esses grupos foram comparados quanto a características socioeconômicas e reprodutivas. O teste estatístico utilizado foi o Qui quadrado de Pearson ${ }^{17}$. Os comentários das mulheres foram transcritos na íntegra para arquivo computadorizado. Foram lidos e relidos pela pesquisadora principal para identificar unidades de significado relativas aos objetivos do estudo ${ }^{18}$. Em seguida, foram agrupadas as unidades semelhantes e categorizadas conforme o seu conteúdo, destacando-se as principais idéias mencionadas em cada categoria.

O protocolo de pesquisa desse estudo foi aprovado pela comissão de pesquisa do departamento de Tocoginecologia e Comitê de Ética em Pesquisa da Faculdade de Ciências Médicas da Universidade Estadual de Campinas.

\section{Resultados}

Foram entregues 420 questionários e devolvidos 378 (perda de 10\%). Entre estes, I I 4 (30\%) continham comentários escritos pelas mulheres que participaram do estudo. A comparação das características das mulheres que escreveram comentários com as que não escreveram mostrou diferença apenas em relação à renda familiar mensal. A presença de comentários foi mais freqüente entre as que referiram menor renda mensal. Não se verificaram diferenças estatisticamente significativas entre os grupos em relação à idade, estado marital, cor da pele, grau de instrução, trabalho remunerado e número de gravidezes. $\mathrm{Na}$ Tabela I são apresentadas as principais categorias de comentários, as idéias analisadas e a freqüência com que essas idéias apareceram.

Nos comentários, as categorias de análise e as principais idéias identificadas foram:

- Insegurança/confusão em relação à vida, a estar ou não na menopausa e às condições de atendimento médico:

..."tem muitas perguntas que nós mulheres queremos fazer a um médico, mas não temos coragem. Deveria ter um questionário em que teríamos a resposta" (47 anos, casada, segundo grau completo, classe socioeconômica D).

..."gostaria de receber mais informações para orientar-me e poder amenizar essa angústia necessária da menopausa" (46 anos, divorciada, segundo grau completo, dois filhos, classe econômica C).

..."Meu tempo já passou. Errei com a minha vida" (52 anos, casada, universitário completo e pós-graduação, quatro filhos, classe socioeconômica B). 
Tabela I - Categorias de análise e freqüência das principais idéias identificadas nos comentários das mulheres ( $n=\mid 14$ )

\begin{tabular}{|c|c|c|}
\hline Categorias & Principais idéias & Freqüência das principais idéias $\mathbf{N}(\%)^{*}$ \\
\hline Sintomas que provocam sentimentos negativos & $\begin{array}{l}\text { Sentir coisas que não sentia; ondas de calor; } \\
\text { ressecamento vaginal; diminuiçãa da disposição; } \\
\text { diminuiçãa da libido; alteração do humor; } \\
\text { dor nas pernas; depressão; não se sentir confortável; } \\
\text { sentir-se frustrada; problemas sem solução; } \\
\text { diminuição dos encantos. }\end{array}$ & $52(45,6 \%)$ \\
\hline Insegurança/confusão & $\begin{array}{l}\text { Sem coragem de fazer perguntas aos médicos; } \\
\text { angústia necessária da menopausa; vida estressada e } \\
\text { cheia de conflitos; errar com a vida; abandono; não sabe } \\
\text { se está na menopausa; necessidade de dar mais atenção à } \\
\text { saúde da mulher; nem tudo é culpa só de mulher; } \\
\text { oportunidade de desabafar; temor; pedido de socorro. }\end{array}$ & $44(38,6 \%)$ \\
\hline Assuntos gerais & $\begin{array}{l}\text { Comentários sobre a pesquisa, eventos que aconteceram } \\
\text { na vida, relatos de problemas ou impressões pessoais etc. }\end{array}$ & $28(24,6 \%)$ \\
\hline Terapiahormonal & $\begin{array}{l}\text { Falta de convicçãa dos médicos; como saber se seu organismo } \\
\text { está com risco aumentado de câncer; insegurança associada ao } \\
\text { medo de câncer e outros efeitos; não gostar do uso de } \\
\text { hormônios; sentir-se cobaia; sem outra opção. }\end{array}$ & $18(15,8 \%)$ \\
\hline
\end{tabular}

*Alguns textos continham mais de uma idéia

..."nessa idade as mulheres têm uma vida estressada e cheia de conflitos, tanto na vida material, ou seja, financeira e também na vida sentimental" (48 anos, desquitada, dois filhos, segundo grau completo, classe socioeconômica D).

..."Eu penso que os profissionais de ginecologia do serviço público devem ter mais treinamento e mais tempo e vagas em suas agendas para dar mais atenção à saúde da mulher nessa faixa etária, pois requer muita atenção e tratamento hormonal" (46 anos, casada, um filho, segundo grau completo, classe socioeconômica D).

..."mesmo tendo confiança na minha ginecologista, fico preocupada se estou na pós-menopausa ou não. A minha preocupação é estar e, não tendo acompanhamento, eu possa ter algum problema" (46 anos, divorciada, segundo grau completo, três filhos, classe socioeconômica E).

Houve também sugestões:

..."Acho que a doutora deveria aproveitar esta pesquisa populacional para debater com companheiros de profissão a respeito do atendimento, principalmente ginecológico, para pessoas carentes, que estão passando por uma fase difícil financeiramente, como eu e outras mais. Uma campanha dessas faria com que muitas mulheres se tornassem mais interessadas em divertir, aproveitar e entender mais do seu próprio corpo. Elas seriam mais felizes e, com isto, viveriam mais" (5I anos, separada, três filhos, segundo grau completo, classe socioeconômica D).

..."Eu acho que deveria ter mais espaço na mídia para falar sobre a saúde da mulher. Outra coisa: deveria ter orientações para os homens. Incentivo para que eles procurassem o médico também. É uma sugestão. Mais informações para os homens procurarem o médico, porque nem tudo é só culpa de mulher" (42 anos, casada, segundo grau completo, dois filhos, classe socioeconômica E).
Essa insegurança/angústia levou as mulheres a fazerem pedidos e desabafos:

..."Socorro, socorro, socorro para nós mulheres rejeitadas aos 50 anos pelos parceiros que vão em busca das únicas molas propulsoras que movem o homem: sexo, visual e dinheiro. Já fomos jovens, mas amamos com o coração. Vem a desilusão, a solidão, o medo de assumir todos os compromissos familiares sozinhas. Pela sociedade também somos ignoradas. E vai a auto-estima rolando escada abaixo. Fico pensando em como reconstruir minha vida numa sociedade que não há mais espaço para mim e neste período tão difícil marcado. Há oscilações hormonais intensas que modificam nosso comportamento a todo momento. Precisamos falar, precisamos urgentemente de grupos de auto-ajuda, como o AA, etc." (5I anos, separada, universitário completo, dois filhos, classe socioeconômica A).

..."no convívio conjugal é muito ruim. É uma fase difícil para o cônjuge. Geralmente ele fica impotente e não conversa sobre o assunto. Prefere resolver seu problema fora, com outras mulheres. Isto acontece com a maioria dos homens. Ele se distancia da mulher tornando-a mais insegura e solitária. É nessa fase que muitos casamentos são desfeitos" (57 anos, casada, segundo grau completo, três filhos, classe socioeconômica A).

- Sintomas que provocam sentimentos negativos. As mulheres escreveram sobre as relações entre a transição climatérica, fatores hormonais e os eventos da vida. A menopausa foi relacionada apenas a perdas:

..."Eu estou na menopausa. Sinto várias coisas que não sentia. Às vezes sinto calor, dor nas pernas, suor demasiado, nervosa, brigo com o marido, não sinto vontade de fazer sexo. Falta de paciência" (50 anos, casada, universitário incompleto, dois filhos, classe socioeconômica B). 
..."Faz um ano que me trato de depressão e esse medicamento tem acabado comigo. Faço controle com a ginecologista a cada seis meses. Perdi muito a lubrificaçãa vaginal. Isso me incomoda muito. Faço uso de gel, mas não me sinto confortável. Sinto-me frustrada. Perdi muito meu orgasmo. Isso me deixa péssima. Fiz recente todos os exames, mas ainda não estou entrando na menopausa. Mas todos esses problemas me preocupam muito. Gostaria muito de ter uma solução para esses problemas que sinto. E tenho poucas informações sobre eles. Gostaria muito que esses assuntos fossem mais divulgados, principalmente da mudança de fase. E mais ainda as doenças e mudanças de sentimentos, comportamentos e quais as soluções" (40 anos, casada, segundo grau completo, três filhos, classe socioeconômica B).

..."Reconheço que hoje tudo é bem diferente. A maciez da pele, dos cabelos, os encantos, a flexibilidade, a disposição e a capacidade de executar muitas tarefas sem o cansaço diminuíram bastante. Com 46 anos, percebo que por mais que a vida esteja cor-de-rosa, os hormônios realmente influenciam e fazem a diferença. Então, o meu relato fica expresso em cima do aumento da irritação, falta de paciência com as crianças, falta de disposiçãa e principalmente na diminuição da libido" (46 anos, casada, universitário completo, dois filhos, classe socioeconômica B).

..."No resultado a médica me informou que eu estava realmente entrando na menopausa. Não sei por que fiquei triste e chateada" (46 anos, divorciada, um filho, segundo grau completo, classe socioeconômica C).

..."Hoje particularmente sinto-me péssima. Estou na TPM. E, socorro, socorro, socorro, parece que a cada mês ela surge com mais intensidade. Ela chega me desequilibrando emocionalmente. Fico totalmente passional, com crises de pânico e medo" (5I anos, separada, universitário completo, dois filhos, classe socioeconômica A).

- Tratamentos: as mulheres se referiram ao tratamento de reposição hormonal mesclando sentimentos de insegurança e medo. Relataram insegurança dos médicos em prescrever ou não uma terapia de reposição hormonal.

..."O que mais me chama a atenção neste período da minha vida é a falta de convicção dos médicos quanto à reposição hormonal. Cada um acha de um jeito e não chegam a conclusão alguma. Uns são a favor, outros são contra e aí fico na dúvida" (57 anos, casada, segundo grau completo, três filhos, classe socioeconômica A).

..."A minha opinião a respeito da terapia de reposição hormonal é que tenho muitas dúvidas e me sinto cobaia. As médicas deixam nas nossas mãos a decisão. Eu optei por fazer porque não consigo conviver com as ondas de calor e o mau humor e, apesar do inchaço e das mamas doloridas causados pelo hormônio, ainda assim me sinto melhor. Espero que as minhas filhas passem por esse período de suas vidas com mais certeza das decisões que tomarem e que tenham melhor qualidade de vida" (50 anos, casada, universitário incompleto, duas filhas, classe socioeconômica A).

..."Acho que o tratamento hormonal deveria ser feito em todas as mulheres, independentemente da conduta médica. Por exemplo: 0 meu ginecologista é contra. Ele acha que em algumas mulheres o organismo predispõe ao câncer. Fazendo a reposiçã̃o, a chance de ter o câncer é maior. Como eu vou saber se o meu organismo é ou não? $\mathrm{Na}$ minha família não existem casos. O tratamento hormonal faz com que minha libido não diminua? Gostaria de saber mais a respeito do assunto" (44 anos, casada, segundo grau completo, três filhos, classe socioeconômica C).

..."Sinto-me insegura quanto à terapia de reposição hormonal. Tenho casos de câncer de mama na família (mãe e tia) e isso me causa inseguranç̧a, pois sei que corro mais riscos" (54 anos, casada, universitário completo, três filhos, classe socioeconômica A).

..."Eu sempre vou ao ginecologista. Falo com ele que estou sem libido, ressecada (vagina). Ele já me deu hormônio, mas ficou do mesmo jeito. Então, pra quê?" (53 anos, casada, segundo grau completo, dois filhos, classe socioeconômica E).

..."Há poucos dias comecei a usar isoflavona. Estou me sentindo melhor. Não gostaria de usar hormônios" (50 anos, casada, universitário incompleto, dois filhos, classe socioeconômica B).

..."Os medicamentos de reposição hormonal naturais são muito caros e eu tenho dois casos de aneurisma cerebral na família e os que não são naturais não são indicados, então fiz opção de não usar e enquanto a menopausa não chega, vou usando produtos que me ajudem na reposição" (52 anos, casada, segundo grau completo, dois filhos, classe socioeconômica D).

\section{Discussão}

O objetivo dessa análise secundária de dados contidos em um projeto maior sobre a sexualidade em mulheres de 40 a 65 anos, residentes em Belo Horizonte, MG'2, foi conhecer a opinião de algumas delas sobre a menopausa e seu tratamento. As que escreveram e as que não o fizeram mostraram que, de maneira geral, não houve associação a uma determinada característica específica. A exceção foi a renda, havendo maior número de comentários entre as mulheres de menor renda familiar mensal. Isso pode ser explicado pela maior carência destas quanto à orientação médica ou de atenção pessoal, representando o questionário uma oportunidade de comunicação, ainda que indireta. Inquérito populacional na cidade de Campinas (SP), constatou que o nível socioeconômico baixo associou-se à não procura de atenção à saúde por mulheres com idades entre 45 e 60 anos ${ }^{19}$. Também é possível pensar que essas mulheres estavam mais predispostas a expor seus sentimentos e angústias no momento de responder ao questionário.

No contexto da insegurança das mulheres quanto ao tratamento de seus sintomas, a afirmação de "não ter coragem de fazer perguntas aos médicos" sugere que esses profissionais podem não ter tempo suficiente ou não estar dispostos a esclarecer e discutir as dúvidas das mulheres durante a consulta, deixando-as inseguras e confusas. Laznik ${ }^{8}$ reforça a importância do ginecologista na clínica de climatério. Se o médico se mostrar atento e pronto para ouvir, a paciente lhe revelará suas aflições.

Observou-se que algumas mulheres revelaram confusão quanto a estar ou não na menopausa e ao relato da perda dos encantos e do interesse do parceiro por elas. Eventos, como a separação conjugal nessa fase, reforçam ainda mais a crise, surgindo o sentimento de rejeição. A mulher, quando se sente desvalorizada pelo parceiro, apresenta a tendência a sentir-se mal. O homem pode, na sua maturidade, sentir-se ameaçado pela perda de fecundidade de sua parceira e ver no corpo dela as marcas do tempo que lhe voltarão, em espelho, como suas. $\mathrm{O}$ poder de estar com uma parceira mais jovem leva em 
muitos casos à sensação de rejuvenescer ${ }^{8}$. George ${ }^{6}$ avaliou I 5 mulheres americanas entre 48 e 62 anos e verificou que muitas delas também expressaram sentimentos de confusão em relação à menopausa. $A$ maioria imaginava-a baseada em anedotas de amigos, informações da mídia e artigos nem sempre confiáveis. Das 15 mulheres pesquisadas, 10 apresentaram alguma forma de labilidade emocional.

Estudo qualitativo realizado na Suécia com mulheres climatéricas descreveu que, embora apresentassem sentimento de liberdade em relação à menopausa, elas associaram o climatério a preocupações e problemas seguidos por diferentes sintomas ${ }^{5}$. Outro estudo 8 mostrou que as mudanças que ocorrem no climatério produzem sentimentos subconscientes associados à imagem da mulher, considerando-se os padrões socioculturais do seu meio. Além disso, essas mudanças podem levar a perda de atributos físicos e alterações dos papéis sociais que foram exercidos até então.

Culpas ou arrependimentos por decisões que levaram a escolhas e vivências anteriores são vistas. Na literatura e na crença de muitos, a menopausa é tratada em bloco, como se fosse preciso associá-la à velhice. Muitas mulheres sentem-se velhas e abandonadas, e com a auto-estima baixa acabam presas a essas percepções ${ }^{8}$.

A literatura é extensa, principalmente em países do Ocidente, e há quase unanimidade nos relatos, evidenciando estar o período do climatério, para muitas mulheres, associado ao medo, dificuldade e ansiedade. Essa visão tão enfática parece ter se confirmado na amostra de mulheres que participou do presente estudo, talvez as com mais sintomas e com mais necessidades de atenção. Segundo Gómez ${ }^{20}$, o principal valor social da mulher, e mesmo a sua identidade, têm sido definidos em termos de seu potencial reprodutivo e de atração. Como conseqüência, para muitas mulheres há dificuldades em aceitar o envelhecimento, sendo o primeiro sinal do término desse potencial procriador a chegada da menopausa, que abre as portas para o desconhecido e, consequentemente, para o tempo.

As mulheres pesquisadas também se mostraram desejosas de informações a respeito da menopausa por meio da mídia. Embora o tema venha ganhando relevância nos debates em saúde, um levantamento dos textos jornalísticos sobre saúde, publicados em revistas de atualidades, constatou que, do total de 384 textos, 13,5\% eram sobre saúde da mulher e 5,2\% sobre menopausa. Observou-se neste estudo a presença de idéias preconceituosas, baseadas em estereótipos de gênero, e o uso do termo menopausa de forma pejorativa. Esclarecer a menopausa nos textos jornalísticos de forma precisa e freqüente pode auxiliar mulheres e homens a compreender melhor essa fase da vida feminina ${ }^{10}$.

Pôde-se constatar, por meio dos comentários analisados, que as mulheres, não conseguindo conviver com os sintomas climatéricos, fizeram uso da terapia hormonal, embora inseguras e superestimando os riscos dessa terapia. Além disso, sentiam-se temerosas em relação ao câncer de mama. George ${ }^{6}$ também registrou que a intervenção farmacológica da menopausa gerou expressiva confusão na maioria das participantes do seu estudo, como conseqüência da falta de informações sobre os riscos e benefícios da terapia hormonal ou de terapias alternativas. Da mesma forma, o medo do câncer de mama foi o exemplo mais importante dessa visão conflituosa. Após a publicação de estudos recentes ${ }^{21,22}$, muitas mulheres suspenderam por decisão própria, ou por orientação médica, o uso da hormonioterapia devido ao pânico global que relacionava essa terapia a problemas com a saúde. No Estado de São Paulo, aproximadamente um quarto dos ginecologistas deixou de prescrever terapia hormonal ${ }^{23}$.

Parece existir carência de conhecimento quanto aos riscos e benefícios da terapia hormonal, tanto pelos médicos como pelas mulheres. Em pesquisa com médicos nos Estados Unidos ${ }^{24}$, encontrouse que a causa de consulta mais freqüentemente relatada por suas clientes foram questões relacionadas à menopausa e ao seu tratamento, e a preocupação mais constante era como tratar esses problemas, em especial os sintomas climatéricos. A seguir, vinham as dúvidas quanto à terapia hormonal. Ainda nessa pesquisa, quando se perguntou aos médicos sobre fitoterápicos, 78\% responderam sentirem-se confortáveis em prescrevê-los. A falsa percepção de que medicamentos naturais são inofensivos e o desconhecimento da falta de eficácia dos mesmos contribuíram para que muitos médicos optassem por essa terapêutica ${ }^{25}$.

\section{Conclusão}

Evidencia-se, portanto, a necessidade de mais investimentos em educação dirigida tanto aos profissionais que assistem essa clientela quanto às mulheres em geral. A mídia poderia contribuir de maneira mais adequada para ampliar o diálogo entre o conhecimento científico e os desejos e necessidades das mulheres nessa importante fase da vida.

Finalmente, cabe ressaltar que, por sua natureza qualitativa, a análise aqui discutida não pode ser generalizada em termos numéricos como sendo representativa das mulheres de Belo Horizonte, MG. Também, cabe lembrar que os comentários analisados não foram obtidos mediante estímulo de uma pergunta específica, mas foram espontâneos. Assim, os resultados discutidos devem ser entendidos como subsídios para aprofundar o conhecimento sobre a vivência e as representações que existem entre as mulheres a respeito da menopausa e do tratamento de seus sintomas. Eles podem contribuir para a melhor compreensão das dificuldades enfrentadas por algumas mulheres em vivenciar a menopausa. Embora não possam ser estatisticamente generalizados, os achados desse estudo apontam para aspectos de profundidade do tema, facilitando o surgimento de idéias que poderão servir de referência para estudos em amostras similares ${ }^{26}$.

\section{Conflito de interesse: não há}

\section{SUMMARY}

THE OPINION OF WOMEN ON MENOPAUSE AND TREATMENT OF ITS SYMPTOMS

OBJECTIVE. To evaluate the perception of a group of women from Belo Horizonte, Minas Gerais, Brasil, with respect to menopause and its treatment.

Methods. A secondary analysis was performed on data from a population-based, cross sectional study carried out with 378 Brazilian born women between 40 and 65 years of age, with II years or more of formal education. Some women added spontaneous comments to the end of the questionnaire on sexuality handed out by research assistants and self-respondedanonymously. There were those who provided comments at 
VALADARES AL ET AL.

the end of the questionnaire and those who did not. The groups were compared with respect to sociodemographic and reproductive characteristics using Pearson's chi-square test. Comments were transcribed in their entirety to a computerized file for thematic content analysis, and units of meaning were identified and classified.

RESULTS. Approximately one-third of the women (1/4/378) provided comments. Significantly more women with lower income levels provided comments as compared to those with higher income levels. The principal identified categories of analysis were: a) uncertainty and/or confusion, mainly with regard to anguish, stress and doubts about menopause; b) symptoms that lead to negative feelings, such as hot flushes, vaginal dryness and mood changes; and c) hormone replacement therapy, fears and perception of a lack of conviction in the physicians who prescribe it.

CONCLUSION. The comments indicate that more attention must be given to the problems perceived in climacteric women with a special emphasis on those of the lower incomes levels. [Rev Assoc Med Bras 2008; 54(4): 299-304]

KeY Words: Climacterium. Hormone replacement therapy. Menopause. Women's health. Cross-sectional studies.

\section{RefERÊNCIAS}

I. Machado LV. Dinâmica hormonal do climatério. In: Endocrinologia ginecológica. Rio de Janeiro: Med Book-Editora Científica Ltda; 2006. p. 197-203

2. Bachmann GA, Leiblum SR. The impact of hormones on menopausal sexuality: a literature review. Menopause. 2004; I I ( I): I 20-30.

3. Melby MK, Lock M, Kaufert P. Culture and symptom reporting at menopause. Hum Reprod Update. 2005; I I (5): 495-5 I 2.

4. Utian WT. Psychosocial and socioeconomic burden of vasomotor symptoms in menopause: a comprehensive review. Health Qual Life Outcomes. 2005;3:47 [cited 2007 Nov 30]. Available from: http:// www.hqlo.com/content/pdf//477-7525-3-47.pdf.

5. Bertero $C$. What do women think about menopause? A qualitative study of women's expectations, apprehensions and knowledge about the climacteric period. Int Nurs Rev. 2003; 50(2): I09- I8.

6. George SA. The menopause experience: a womans perspective. J Obstet Gynecol. Neonatal Nurs. 2002;3 I ( I):77-85.

7. Dennerstein L, Randolph J, Taffe J, Dudley E, Burger H. Hormones, mood, sexuality, and the menopausal transition. Fertil Steril. 2002;77(4):42-8

8. Lasnick MC. O complexo de Jocasta: feminilidade e sexualidade pelo prisma da menopausa. Rio de Janeiro: Companhia de Freud; 2004.

9. Hunter M, O'Dea I. An evaluation of a health education intervention for mid-aged women: five year follow-up of effects upon knowledge, impact of menopause and health. Patient Educ Couns. 1999;38(3):249-55.
10. Amaral ICGA. Abordagem da menopausa em textos jornalísticos veiculados em revistas de atualidades [dissertação] Campinas: Faculdade de Ciências Médicas, Universidade Estadual de Campinas; 2004

I I. Pinto-Neto AM, Pedro AO, Hardy E, Osis MJD, Costa-Paiva LHS, Martinez EZ. Caracterização das usuárias de terapia de reposição hormonal do Município de Campinas, São Paulo. Cad Saude Publica. 2002; I 8(I ): I 2 I -7.

12. Valadares AL, Pinto-Neto AM, Osis MJ, Conde DM, Sousa MH, Costa-Paiva L. Sexuality in Brazilian women aged 40 to 65 years with I I years or more of formal education: associated factors. Menopause. 2008; I 5:264-9.

13. Instituto Brasileiro de Geografia e Pesquisa (IBGE). Recenseamento geral da população; 2000. Rio de Janeiro: IBGE Editora; 2000.

14. Laumann EO, Paik AMA, Rosen RC. Sexual dysfunction in the United States: prevalence and predictors. JAMA. 1999;28I(6):537-44.

15. Dennerstein L, Lehert P, Dudley E. Short scale to measure female sexuality: adapted from McCoy female sexuality questionnaire. J Sex Marital Ther. 2001;27(4):339-51.

16. Associação Brasileira de Empresas de Pesquisa. Critério de Classificação Econômica Brasil. [citado 2005 Mar 10]. Disponível em: http:// www.abep.org/codigosguias/ABEP CCEB.pdf.

17. Altman DG. Practical statistics for mēdical research. Boca Raton: Chapman \& Hall/CRC; 1999

18. Minayo MCS. O desafio do conhecimento: pesquisa qualitativa em saúde. $2 a$ ed. São Paulo: Hucitec-Abrasco; 1993. p.48.

19. Pedro AO, Pinto-Neto AM, Costa Paiva LHS, Osis MJ, Hardy E. Procura de serviço médico por mulheres climatéricas brasileiras. Rev Saude Publica. 2002;36(4):484-90.

20. Gomez A. La revolucion de las canas: reflexiones y experiências sobre el envejecer de las mujeres. Red de salud de las mujeres latino americanas y del Caribe. Cuad Mujer Salud 1999;4( I):2-8.

21. Rossouw JE, Anderson GL, Prentice RL, LaCroix AZ, Kooperberg C, Stefanick ML, et al. Risks and benefits of estrogen plus progestin in healthy postmenopausal women: principal results from the Womens Health Initiative randomized controlled trial. JAMA. 2002;288(3):32I-33.

22. Hoffmann M, Hammar M, Kjellgreen KI, Lind-Astrand L, Brynhildsen J. Changes in women's attitudes towards and use of hormone therapy after HERS and WHI. Maturitas. 2005;52( I): I I-7.

23. Lazar F Jr, Costa-Paiva L, Morais SS, Pedro AO, Pinto-Neto AM. The attitude of gynecologists in São Paulo, Brazil 3 years after the Womens Health Initiative study. Maturitas. 2007;56(2): I 29-4I.

24. Singh B, Liu XD, Der-Martirosian C, Hardy M, Singh V, Shepard N, et al. A national probability survey of American Medical Association gynecologists and primary care physicians concerning menopause. Am J Obstet Gynecol. 2005; 193(3 Pt I):693-700.

25. Krebbs EE, Ensrud KE, McDonald R, Wilt TJ. Phytoestrogens for treatment of menopausal symptoms: a systematic review. Menopause. 2004; 104(4):824-36.

26. Morse JM. Strategies for sampling. In: Morse JM. Qualitative nursing research: a contemporary dialogue. Newbury Park: Sage; 1999. p. 127-56.

Artigo recebido: 02/12/07

Aceito para publicação: 26/03/08 